\title{
3-D electromagnetic induction studies using the Swarm constellation: Mapping conductivity anomalies in the Earth's mantle
}

\author{
Alexei Kuvshinov ${ }^{1 *}$, Terence Sabaka ${ }^{2}$, and Nils Olsen ${ }^{1}$ \\ ${ }^{1}$ Danish National Space Center, Juliane Maries Vej 30, DK-2100 Copenhagen, Denmark \\ ${ }^{2}$ Geodynamics Branch, NASA GSFC, Greenbelt/MD, USA
}

(Received November 19, 2004; Revised August 25, 2005; Accepted September 28, 2005; Online published April 14, 2006)

\begin{abstract}
An approach is presented to detect deep-seated regional conductivity anomalies by analysis of magnetic observations taken by low-Earth-orbiting satellites. The approach deals with recovery of $C$-responses on a regular grid and starts with a determination of time series of external and internal coefficients of the magnetic potential. From the coefficients, time series of the magnetic vertical component and of the horizontal divergence of the horizontal components are synthesized on the grid and the $C$-responses are determined by means of signal processing of the corresponding time series. For validation of the approach, 3 years of realistic synthetic data at simulated orbits of the forthcoming Swarm constellation of 3 satellites have been used. To obtain the synthetic data for a given 3-D conductivity Earth's model a time-domain scheme has been applied which relies on a Fourier transformation of the inducing field, and on a frequency domain forward modelling. The conductivity model consists of a thin surface layer of realistic conductance and a 3-D mantle that incorporates a hypothetic deep regional anomaly beneath the Pacific Ocean plate. To establish the ability of the approach to capture the geometry of the mantle heterogeneities used in the forward approach, numerical experiments have been undertaken using various satellite combinations, sampling periods of the resulting time series, and numbers of internal coefficients. The possibility of the approach to map anomalies in the mantle using satellite data that contain contributions from the core and lithosphere, from the magnetosphere and ionosphere (and their Earth-induced counterparts), as well as payload noise has been investigated. The model studies have shown that $C$-responses obtained on a regular grid might be used to map regional deep-seated conductivity anomalies. Moreover, it has been demonstrated that these $C$-responses are successfully recovered from magnetic data collected by the proposed Swarm constellation of 3 satellites.
\end{abstract}

Key words: Satellite magnetic observations, 3-D electromagnetic induction, $C$-responses, mantle conductivity anomalies.

\section{Introduction}

The study of lateral variability in physical properties of Earth's mantle using geophysical methods is a topic of modern fundamental science as it gives insight into geodynamic processes such as mantle convection, the fate of subducting slabs and the origin of continents. Global seismic tomography (cf. Li and Romanowicz, 1995; Woodhouse and Trampert, 1995; Su and Dziewonski, 1997; Ritsema et al., 1999; Bijwaard and Spakman, 2000; Deschamps et al., 2002) provides today a variety of three-dimensional (3-D) mantle velocity models which can be interpreted in terms of cratonic roots, mantle plumes and slab graveyards.

The goal of electromagnetic (EM) induction studies is to identify complementary large-scale spatial variations (3-D structures) in the electrical conductivity of the mantle. This is an important issue since conductivity reflects the connec-

\footnotetext{
${ }^{*}$ On leave from Institute of Terrestrial Magnetism, Ionosphere and Radiowave Propagation, Russian Academy of Sciences, 142190, Troitsk, Moscow region, Russia.

Copyright (c) The Society of Geomagnetism and Earth, Planetary and Space Sciences (SGEPSS); The Seismological Society of Japan; The Volcanological Society of Japan; The Geodetic Society of Japan; The Japanese Society for Planetary Sciences; TERRAPUB
}

tivity of constituents as graphite, fluids, partial melt, and volatiles (all of which may have profound effects on rheology and, eventually, mantle convection and tectonic activity), while seismology ascertains bulk mechanical properties.

Traditionally, ground-based observatory recordings of the geomagnetic field (cf. Roberts, 1984; Schultz and Larsen, 1987; Schultz, 1990; Olsen, 1998) along with longperiod magnetotelluric (MT) measurements (cf. Egbert and Booker, 1992; Schultz et al., 1993; Neal et al., 2000; Semenov et al., 2003), and submarine cable recordings of voltage differences (cf. Lizarralde et al., 1995; Fujii and Utada, 2000; Utada et al., 2003; Santos et al., 2003) have been used to detect radial mantle conductivity variations. But global images of 3-D mantle heterogeneities can hardly be obtained with the use of ground-based data due to the sparse and very irregular distribution of geomagnetic observatories and long-period MT sites. For instance, the about $75 \%$ of the globe that are occupied by oceans is almost free of geomagnetic observatories and MT sites. Voltage data from transoceanic submarine cables somehow fill this gap (cf. Fukao et al., 2004) but, again, cables are rare and provide only an integral characteristic (voltage), making it impossible to trace lateral variations of conductivity along the cable. 

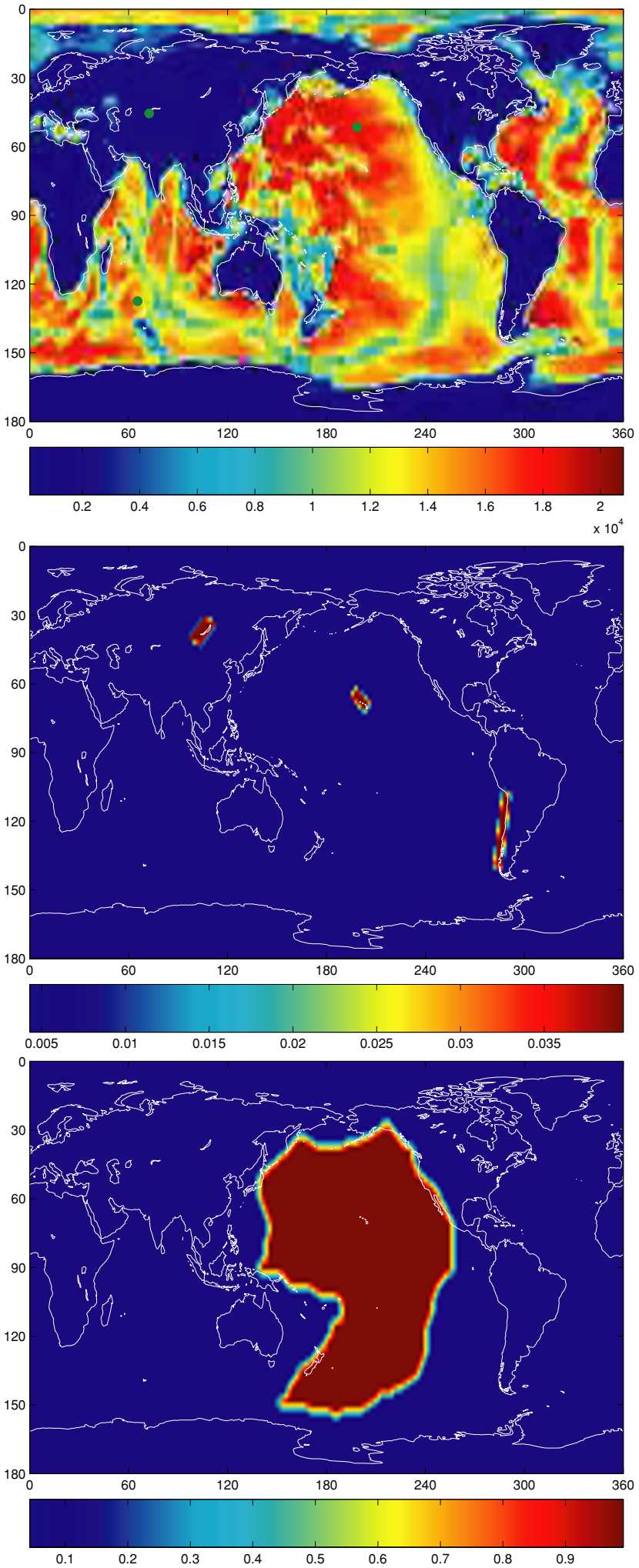

Fig. 1. Top: Surface shell conductance in units of S. Middle: Conductivity [S/m] at depths from $1 \mathrm{~km}$ down to $400 \mathrm{~km}$. Bottom: Conductivity [S/m] at depths from $400 \mathrm{~km}$ down to $700 \mathrm{~km}$.

Satellite-borne measurements provide an intriguing and unique source of improving our knowledge about 3-D variations of electrical conductivity in the Earth's mantle owing to a good spatial coverage with high-precision data of uniform quality. However, satellites move with a speed of a few $\mathrm{km}$ per second and thus measure some mixture of temporal and spatial changes of the magnetic field. This makes satellite data more difficult to process compared to ground data. In spite of this problem several successful attempts have been made to derive a global conductivity-depth (1-D) distribution from magnetic satellite measurements (cf. Didwall, 1984; Oraevsky et al., 1993; Olsen, 1999; Olsen et al., 2002; Constable and Constable, 2004). On the other hand, until now 3-D induction studies with satellite data are confined to simulating magnetic effects of conductivity anomalies at satellite altitudes (cf. Kuvshinov et al., 1998; Olsen, 1999; Tarits and Grammatica, 2000; Grammatica and Tarits, 2002; Everett et al., 2003; Velimsky et al., 2003; Kuvshinov and Olsen, 2005b; McCreadie and Martinec, 2005; Velimsky and Everett, 2005).

In this paper we make a first attempt to map deep 3-D anomalies from space. Our analysis deals with a recovery of global maps of $C$-responses by processing realistic signals calculated in the frame of the closed-loop simulation of the Swarm multi-satellite mission (cf. Olsen et al., 2006).

The contents of the paper is as follows. Section 2 describes the 3-D conductivity model that has been designed for our simulation studies. Section 3 presents the timedomain scheme that was applied to produce synthetic induced magnetic signals from a given magnetospheric source distribution using this 3-D conductivity model at the orbits of the Swarm constellation. Transfer functions $(C$ responses) for induction studies are discussed in Section 4. Model studies to validate the ability of $C$-responses to capture the geometry of the mantle inhomogeneities are presented in Section 5. Section 6 introduces a scheme for recovering these $C$-responses from satellite data and presents results obtained with data that only contain contribution due to magnetospheric sources. Section 7 reports on $C$-response determination using data that comprise, along with magnetospheric signals, realistic contributions from all other sources modelled - from core and crustal fields, ionospheric variations, as well as payload noise. Conclusions and some suggestions for further work are presented in Section 8.

\section{Design of the 3-D Conductivity Model}

The 3-D conductivity model that we consider consists of an inhomogeneous conducting surface shell, three local conductors of $0.04 \mathrm{~S} / \mathrm{m}$ at depths from the bottom of that shell down to $400 \mathrm{~km}$, and a deep-seated regional conductor of $1 \mathrm{~S} / \mathrm{m}$ located between $400 \mathrm{~km}$ and $700 \mathrm{~km}$ depth. The local and regional conductors are embedded in a radially symmetric section consisting of a relatively resistive $400 \mathrm{~km}$ thick layer of $0.004 \mathrm{~S} / \mathrm{m}$, a $300 \mathrm{~km}$ thick transition layer of $0.04 \mathrm{~S} / \mathrm{m}$, and an inner uniform sphere of $2 \mathrm{~S} / \mathrm{m}$.

Figure 1 shows global maps of these anomalous structures. The top panel presents the adopted surface shell conductance. It approximates the nonuniform distribution of oceans and continents. The conductance of the shell is chosen as realistic as possible and includes contributions from sea water and from sediments. The conductance of the oceans has been derived from the global $5^{\prime} \times 5^{\prime}$ NOAA ETOPO map of bathymetry, multiplying the water depth by a mean seawater conductivity of $3.2 \mathrm{~S} / \mathrm{m}$. The conductance of the sediments has been derived from the global sediment 


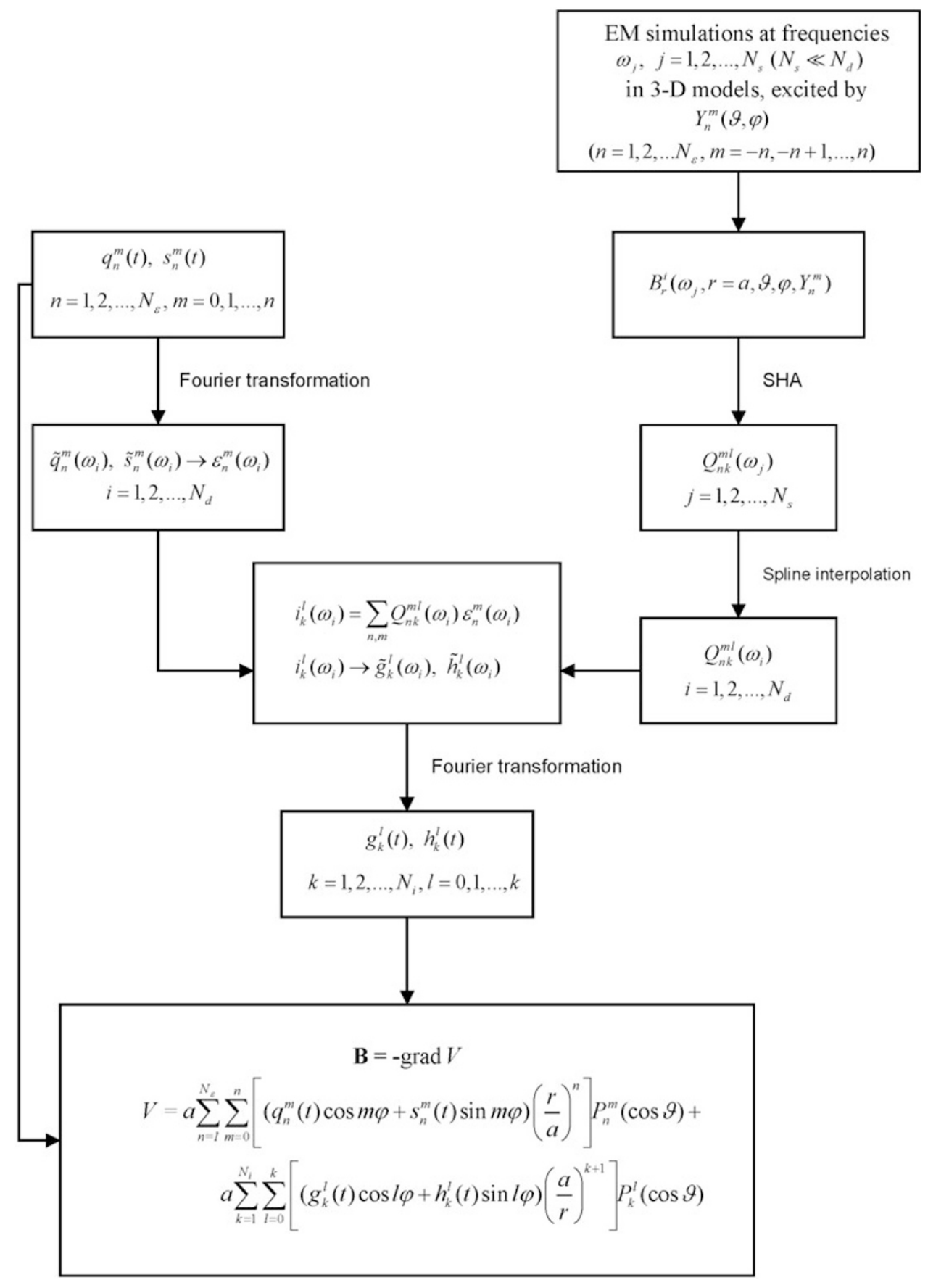

Fig. 2. Scheme describing the calculation of induced field contributions.

thickness given by the $1^{\circ} \times 1^{\circ}$ map of Laske and Masters (1997) with the use of a heuristic procedure similar to that described in Everett et al. (2003).

Two local conductors of horizontal size $600 \times 1200 \mathrm{~km}^{2}$ describe hypothetic plums beneath the Baikal and Hawaii (Constable and Heinson, 2004); the third conductor of size of $600 \times 3000 \mathrm{~km}^{2}$ represents a hypothetic subduction zone along the western margin of South America. This part of the model is shown in the middle panel of the Figure and was suggested by Steve Constable (2003; private communication). Finally, the deep-seated large scale structure describing a hypothetic conductor beneath the Pacific Ocean plate is presented in the bottom panel of the figure. The aim of this model is not to be identical to the "true" world (e.g., we do not claim that there really exists a plume under the Baikal), but to provide a test model for our retrieval algorithm. The model was split in the vertical direction into 4 inhomogeneous spherical sublayers of thickness 1, 150, 250 , and $300 \mathrm{~km}$, respectively; each spherical sublayer was discretized in horizontal direction in $180 \times 90$ cells of size $2^{\circ} \times 2^{\circ}$.

\section{3-D Time-Domain Modeling of Swarm Satellite Signals Induced by Magnetospheric Sources}

The procedure shown in Fig. 2 has been used to produce magnetic fields due to magnetospheric sources at the orbits of the Swarm satellites for a given 3-D spherical conductivity model of the Earth and for a given time series of hourly 
mean values of external (inducing) coefficients $q_{n}^{m}(t)$ and $s_{n}^{m}(t)\left(n=1,2, \ldots, N_{\varepsilon}, m=0,1, \ldots, n\right)$ of the magnetic potential (details of derivation of inducing coefficients are presented in the companion paper of Olsen et al. (2006)). This procedure follows in general the scheme described in Olsen and Kuvshinov (2004) and Kuvshinov and Olsen (2005b) and consists of the following steps

1) The time series of the external coefficients $q_{n}^{m}(t)$ and $s_{n}^{m}(t)$ are Fourier transformed to obtain complex coefficients $\tilde{q}_{n}^{m}(\omega)$ and $\tilde{s}_{n}^{m}(\omega)$ at a set of frequencies, $\omega_{i}, i=1,2, \ldots, N_{d}$, where $N_{d}=\frac{P}{2 \Delta t}$. By performing discrete Fourier transform of a time series of external coefficients on an interval $[0, P]$ we implicitly assume that our time series are represented by a finite trigonometric series. Due to the quasi-regular nature of the magnetospheric signal at a long time scale (in our case $P=5$ years) we believe that such a representation is accurate enough for our time-domain simulations.

2) EM induction simulations are performed using the above described 3-D model of electrical conductivity in the frequency domain for $N_{s}$ logarithmically spaced frequencies, $\omega_{j}, j=1,2, \ldots N_{s}$, covering the frequency range from $\omega_{1}=\frac{2 \pi}{P}$ to $\omega_{N_{s}}=\frac{2 \pi}{2 \Delta t}$ (here $\Delta t=1$ hour). For each frequency the simulations are performed for a set of preselected elementary harmonics, $Y_{n}^{m}(\vartheta, \varphi)=P_{n}^{|m|}(\cos \vartheta) e^{i m \varphi}$ of the external field (in our case for all harmonics up to degree $N_{\varepsilon}=3$ ). To simulate the magnetic fields the frequency domain 3D numerical solution (Kuvshinov et al., 2002, 2005), which is based on an integral equation approach, is used. Note that there is no need to calculate the fields at all involved frequencies, $\omega_{i}$, of the inducing field. Due to the smoothed (with respect to frequency) nature of the induced field we calculate the response at a coarse grid of frequencies, $\omega_{j}$ (with 9 frequencies per frequency decade), with subsequent interpolation to all frequencies (see Step 4).

3) For each elementary harmonic, $Y_{n}^{m}(\vartheta, \varphi) \quad(n=$ $\left.1,2, \ldots, N_{\varepsilon}, m=-n,-n+1, \ldots, n\right)$ and each frequency, $\omega_{j}$, a spherical harmonic analysis of the simulated induced part of $B_{r}$ (from step 2) is performed, resulting in arrays of coefficients of the induced part of the potential, $Q_{n k}^{m l}\left(\omega_{j}\right)$ for all harmonics up to degree $N_{i}$ (where $N_{i}$ is determined from the chosen horizontal discretization of the 3-D model. Here $N_{i}=45$ ). $Q_{n k}^{m l}\left(\omega_{j}\right)$ is the field of degree $k$ and order $l$ that is induced by a magnetospheric source, $Y_{n}^{m}(\vartheta, \varphi)$, of degree $n$ and order $m$.

4) The arrays $Q_{n k}^{m l}\left(\omega_{j}\right)$ are spline interpolated from the coarse logarithmically-spaced frequency set $\omega_{j}$ to the actual (denser) frequency set $\omega_{i}$, and the resulting coefficients arrays $\iota_{k}^{l}\left(\omega_{i}\right)\left(k=1,2, \ldots, N_{i}, \quad l=\right.$ $-k,-k+1, \ldots, k)$ are calculated as (Olsen, 1999)

$$
\iota_{k}^{l}\left(\omega_{i}\right)=\sum_{n=1}^{N_{\varepsilon}} \sum_{m=-n}^{n} Q_{n k}^{m l}\left(\omega_{i}\right) \varepsilon_{n}^{m}\left(\omega_{i}\right),
$$

where

$$
\varepsilon_{n}^{m}= \begin{cases}\frac{\tilde{q}_{n}^{m}-i \tilde{s}_{n}^{m}}{\left.\tilde{q}_{n}^{\mid m}\right|^{2}+i \tilde{s}_{n}^{|m|}}, & m>0 \\ \tilde{q}_{n}^{m}, & m<0 \\ & m=0\end{cases}
$$

This step gives frequency domain coefficients of the induced part of the potential produced by given external coefficients $\varepsilon_{n}^{m}$.

5) The complex coefficients $\iota_{k}^{l}\left(\omega_{i}\right)$ are transformed to the real coefficients $\tilde{g}_{k}^{l}\left(\omega_{i}\right)$ and $\tilde{h}_{k}^{l}\left(\omega_{i}\right) \quad(k=$ $\left.1,2, \ldots, N_{i}, l=0,1, \ldots, k\right)$ as $\tilde{g}_{k}^{l}=\iota_{k}^{-l}+\iota_{k}^{l}$ and $\tilde{h}_{k}^{l}=-i\left(\iota_{k}^{-l}-\iota_{k}^{l}\right)$ for $l \neq 0$, and $\tilde{g}_{k}^{l}=\iota_{k}^{l}$ for $l=0$. Then, the coefficients $\tilde{g}_{k}^{l}(\omega)$ and $\tilde{h}_{k}^{l}(\omega)$ are Fourier transformed to the time domain, resulting in time series of hourly values of the coefficients $g_{k}^{l}(t)$ and $h_{k}^{l}(t)$ of the induced part of the potential.

6) The obtained hourly values of coefficients of external and induced parts of the potential, $q_{n}^{m}, s_{n}^{m}$ and $g_{k}^{l}, h_{k}^{l}$, are linearly interpolated to the time instants (sampling interval of $1 \mathrm{~min}$ and $5 \mathrm{sec}$, respectively) of the Swarm satellite positions.

7) Finally the magnetic field $\mathbf{B}=-\nabla V$ at the position of the Swarm satellites is obtained from the scalar magnetic potential $V$, which is approximated by the spherical harmonic expansion

$$
\begin{aligned}
V=a \sum_{n=1}^{N_{\varepsilon}} \sum_{m=0}^{n}[ & \left(q_{n}^{m}(t) \cos m \varphi\right. \\
& \left.\left.+s_{n}^{m}(t) \sin m \varphi\right)\left(\frac{r}{a}\right)^{n}\right] P_{n}^{m}(\cos \vartheta) \\
+a \sum_{k=1}^{N_{i}} \sum_{l=0}^{k}[ & {\left[\left(g_{k}^{l}(t) \cos l \varphi\right.\right.} \\
& \left.\left.+h_{k}^{l}(t) \sin l \varphi\right)\left(\frac{a}{r}\right)^{k+1}\right] P_{k}^{l}(\cos \vartheta)
\end{aligned}
$$

with $a=6371.2 \mathrm{~km}$ as the mean Earth's radius, $\vartheta$ and $\varphi$ as geographic colatitude and longitude and $P_{n}^{m}, P_{k}^{l}$ as associated Legendre functions.

The most complicated and time consuming part of the scheme is the frequency-domain 3-D EM simulations (step 2). Each simulation on an adopted mesh $N_{r} \times N_{\vartheta} \times N_{\varphi}=$ $4 \times 90 \times 180$ (for a given frequency and elementary spherical harmonic) takes about $30 \mathrm{CPU}$ minutes on $2.1 \mathrm{GHz} \mathrm{PC}$.

Note, however, that once the simulations have been performed for a given 3-D model, one can calculate magnetic signals induced in the specific 3-D model for any constellation scenario and any external excitation.

The magnetic field at the position of the Swarm satellites, as well as time series of the coefficients $q_{n}^{m}(t), s_{n}^{m}(t)$, and $g_{k}^{l}(t), h_{k}^{l}(t)$ are used in subsequent sections to infer global maps of $C$-responses.

\section{4. $C$-responses}

Conventionally, the geomagnetic depth sounding (GDS) method (Schmucker, 1985) is used to infer 1-D mantle 

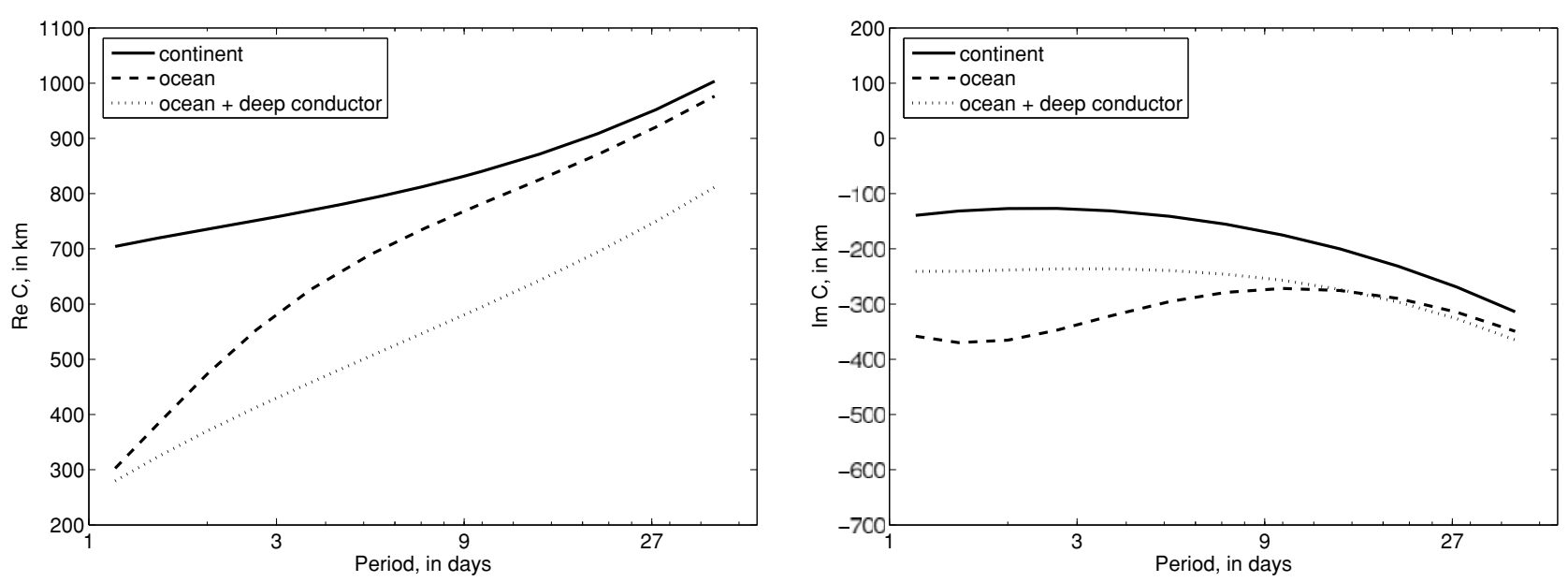

Fig. 3. Real (left panel) and imaginary (right panel) parts of $C$-responses calculated for local 1-D conductivity profiles from three different regions of the constructed 3-D Earth model.
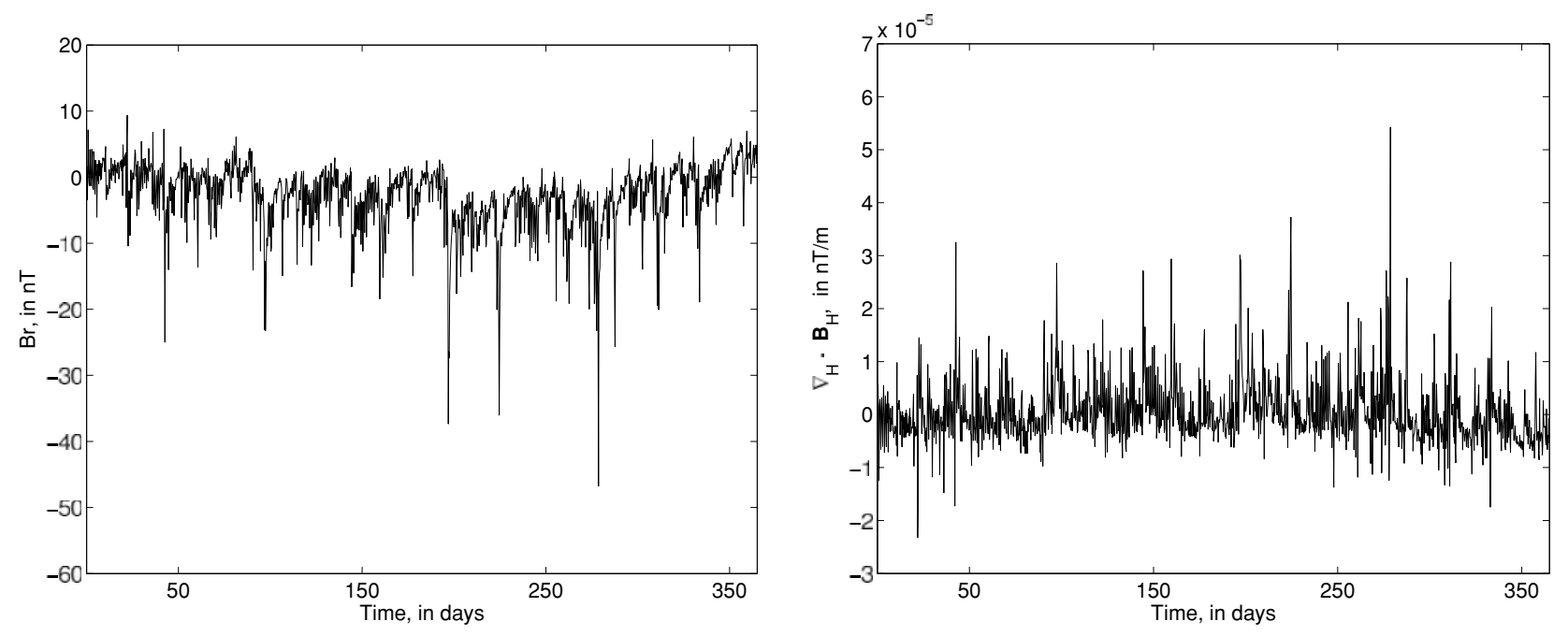

Fig. 4. Time series of $B_{r}$ (left panel) and $\nabla_{H} \cdot \mathbf{B}_{H}$ (right panel) for year 2000, obtained at a site located in Central Eurasia.

conductivity-depth profiles and to detect lateral variations of conductivity. The GDS method is based on the determination and interpretation of response functions like $C$, which is defined as

$$
C(\omega, r, \vartheta, \varphi)=-\frac{B_{r}(\omega, r, \vartheta, \varphi)}{\nabla_{H} \cdot \mathbf{B}_{H}(\omega, r, \vartheta, \varphi)}
$$

with

$$
\nabla_{H} \cdot \mathbf{B}_{H}(\omega, r, \vartheta, \varphi)=\frac{1}{r \sin \vartheta}\left\{\frac{\partial\left(B_{\vartheta} \sin \vartheta\right)}{\partial \vartheta}+\frac{\partial B_{\varphi}}{\partial \varphi}\right\}
$$

being the horizontal divergence of the horizontal component, $\mathbf{B}_{H}$. If the conductivity of the Earth has a radiallysymmetric distribution and the excitation current is of large scale (i.e., described by low degree spherical harmonics), the $C$-response is asymptotically independent of the source geometry (cf. Schmucker, 1985) and depends only on frequency and the conductivity distribution of the Earth. In this case, $|C|$ can be considered as a measure of the "effective" depth of penetration of the EM field into the conducting Earth. Determining $C$-responses at a number of frequencies (by means of signal processing of the corresponding time series of $B_{r}$ and $\nabla_{H} \cdot \mathbf{B}_{H}$ at specific sites) allows for the determination of conductivity-depth profiles (cf. Olsen, 1998) beneath these sites. Recognizing that the periods used in the GDS method typically span a few hours to months, the derived $C$-responses probe upper and midmantle conductivities in the depth range of 100-1000 km or so. As an example, Fig. 3 shows the real (left panel) and imaginary (right panel) parts of the $C$-responses in the period range between 1 and 39 days for three sites with different conductivity vs. depth profiles beneath the sites (the location of these sites are shown in the upper panel of Fig. 1 by green circles). Note, that these $C$-responses come from a 1-D quasi-analytic solution. The solid curves represent $C$-responses for a continental 1-D conductivity profile, whereas the dotted and dashed curves show responses for $1-D$ conductivity profiles in oceanic regions with and without the deep-seated conductor, respectively. As can be seen from the Figure, the maximum difference between continental and oceanic responses is at the shortest considered period ( 1 day), where the relative difference between the responses reaches $130 \%$. It is also seen that the real, $\operatorname{Re}\{C\}$, 

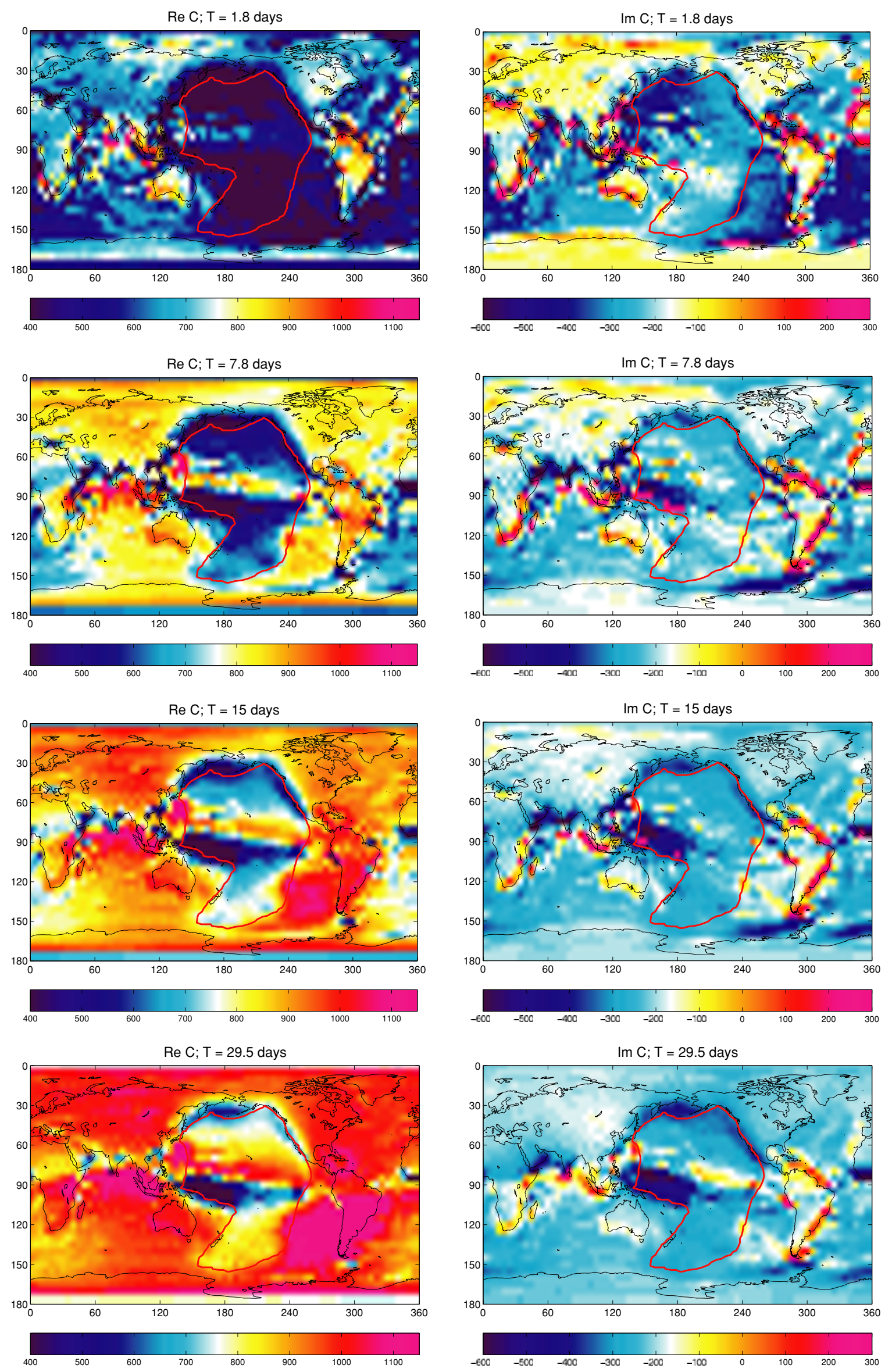

Fig. 5. Real (left panels) and imaginary (right panels) parts of reference $C$-responses at periods of 1.8, 7.8, 15 and 29.5 days (from the top to the bottom), obtained from given time series $q_{n}^{m}(t), s_{n}^{m}(t)$ and $g_{k}^{l}(t), h_{k}^{l}(t)$. 
and imaginary, $\operatorname{Im}\{C\}$, parts of the oceanic $C$-responses show different behaviour concerning the inclusion of the deep conductor in the oceanic 1-D profile. At a period of 1 day the manifestation of the deep conductor in $\operatorname{Re}\{C\}$ is weak; $\operatorname{Re}\{C\}$ of the oceanic 1-D profiles with and without the deep conductor almost coincide. At longer periods $\operatorname{Re}\{C\}$ of the oceanic 1-D profile without the deep conductor reaches that of the continental site: at a period of 14 days their difference does not exceed $7 \%$. With increasing period, the relative difference between $\operatorname{Re}\{C\}$ from $1-\mathrm{D}$ profiles with and without a deep conductor increases, reaching a maximum value of $40 \%$ (compared with $\operatorname{Re}\{C\}$ of the oceanic profile with deep conductor) at a period of 7 days. In general, $\operatorname{Re}\{C\}$ is-in the considered period range (a few days or more) —at least three times larger compared to $\operatorname{Im}\{C\}$, and more sensitive to the presence of the deep conductor.

These 1-D results illustrate that if one succeeds in determining $C$-responses on some regular grid at the surface of the Earth, it is quite plausible to suppose that the deep conductor (see lower panel of Fig. 1) might be seen in the resulting $C$-responses maps at periods larger than a few days. It should be stressed, however, that the results depicted in Fig. 3 are obtained using 1-D conductivity models excited by a source geometry given by the first zonal harmonic, $P_{1}^{0}$.

Another remark to be made is that for the existing sparse and irregular distribution of observatories it is difficult to determine the horizontal field gradients accurately, at least on a global scale. Only satellites have the potential to accomplish this. However, since satellites measure a mixture of temporal and spatial changes of the magnetic field, the recovery of $C$-response from satellite data is a nontrivial task.

\section{Deriving the Reference $C$-responses}

In this section we derive the reference (also called the "true") $C$-responses from given time series $q_{n}^{m}(t), s_{n}^{m}(t)$ and $g_{k}^{l}(t), h_{k}^{l}(t)$ of external and internal expansion coefficients, respectively (recovering these time series from Swarm observations will be discussed in the next sections). First we calculate time series of $B_{r}$ and $\nabla_{H} \cdot \mathbf{B}_{H}$ on a regular grid of $5^{\circ} \times 5^{\circ}$ resolution (this grid will be used in all presented model studies) using the following equation for $\nabla_{H} \cdot \mathbf{B}_{H}$

$$
\begin{aligned}
\nabla_{H} \cdot \mathbf{B}_{H}=\frac{1}{r} \sum_{n=1}^{N_{\varepsilon}} \sum_{m=0}^{n}[ & n(n+1)\left(q_{n}^{m}(t) \cos m \varphi\right. \\
& \left.\left.+s_{n}^{m}(t) \sin m \varphi\right)\left(\frac{r}{a}\right)^{n-1}\right] P_{n}^{m}(\cos \vartheta) \\
+\frac{1}{r} \sum_{k=1}^{N_{i}} \sum_{l=0}^{k}\left[\begin{array}{l}
k(k+1)\left(g_{k}^{l}(t) \cos l \varphi\right. \\
+
\end{array}\right. & \left.\left.h_{k}^{l}(t) \sin l \varphi\right)\left(\frac{a}{r}\right)^{k+2}\right] P_{k}^{l}(\cos \vartheta) .
\end{aligned}
$$

The sampling interval, $\Delta_{C}$, and the length of the time series, $L$, were chosen to be 6 hours and 3 years (1999-2002) respectively. To demonstrate the complexity and realism of the signals, Figure 4 presents time series of $B_{r}$ (left panel) and $\nabla_{H} \cdot \mathbf{B}_{H}$ (right panel) for the year 2000, obtained at a site located in Central Eurasia (shown in the upper panel of Fig. 1 by a green circle).

After signal processing the time series of $B_{r}(t)$ and $\nabla_{H} \cdot \mathbf{B}_{H}(t)$ (using the scheme presented by Olsen (1998)), $C$-responses have been estimated on a regular grid for periods between 1.8 and 29.5 days. By estimating $C$-responses we follow the conventional approach that is based on the processing of continuous time-series without discriminating between storm-time and quiet-time intervals. Moreover, since we work with realistic but still synthetic data (which, in particular, means that the magnetospheric signal has been successfully isolated from ionospheric contribution), it is not so important to distinguish between day-time and nighttime data subsets. Figure 5 shows maps of the real and imaginary parts of $C$ for periods of $1.8,7.8,15$, and 29.5 days. The anomalous behavior of the $C$-responses near the magnetic equator is due to the fact that $B_{r}$ as well as $\nabla_{H} \cdot \mathbf{B}_{H}$ are close to zero here (since source geometry is dominated by $P_{1}^{0}$ in geomagnetic coordinates), which makes the estimation of $C$-responses unstable in this region. The squared coherency of time series $B_{r}(t)$ and $\nabla_{H} \cdot \mathbf{B}_{H}(t)$ are below 0.6 here, which means that the response functions in this region should either be rejected or taken with extreme caution. Nevertheless the figure undoubtedly demonstrates that the deep regional structure beneath the Pacific plate can be detected and mapped. The most prominent manifestation of the anomaly (the geometry of which is shown by the red line) is observed in $\operatorname{Re}\{C\}$ at a period of 7.8 days, with gradual decay of the effect towards longer periods. It is also seen that at a period of 1.8 days the deep regional anomaly is masked by the ocean.

These results have been obtained using all $N_{i}\left(N_{i}+2\right)$, $N_{i}=45$ internal coefficients. However, it is probably difficult to resolve coefficients up to such a high degree. Figure 6 presents maps of $C$-responses at a period of 7.8 days obtained using different truncation levels of the induced fields when constructing $B_{r}$ and $\nabla_{H} \cdot \mathbf{B}_{H}$. The upper and lower panels present maps for maximum degree, $N_{i}$, of 9 and 5, respectively. Decreasing $N_{i}$ from 45 to 9 yields results that are rather close to those obtained with all coefficients (cf. second from the top panels for a period of 7.8 days on Fig. 5). Even for $N_{i}=5$ the regional anomaly is clearly identified in the $C$-responses.

There is no manifestation of the local subsurface conductors (plums and subduction zone; cf. middle panel of Fig. 1) in the derived $C$-responses. The main reason for this is that it is difficult to resolve structures in the depth range between a few to a few hundred $\mathrm{km}$ with the periods in consideration.

\section{6. $C$-responses from Satellite Data that Contain Only Magnetospheric Contribution}

In this section we present results obtained from satellite data that contain only magnetospheric primary and induced fields, but no other contributions (such as from the core field, lithosphere field, ionospheric variations as well as payload noise). The aim of these studies is to investigate whether one can recover $C$-response maps from single- or multiple-satellite data which are consistent with the true $C$ responses obtained in the previous section. 

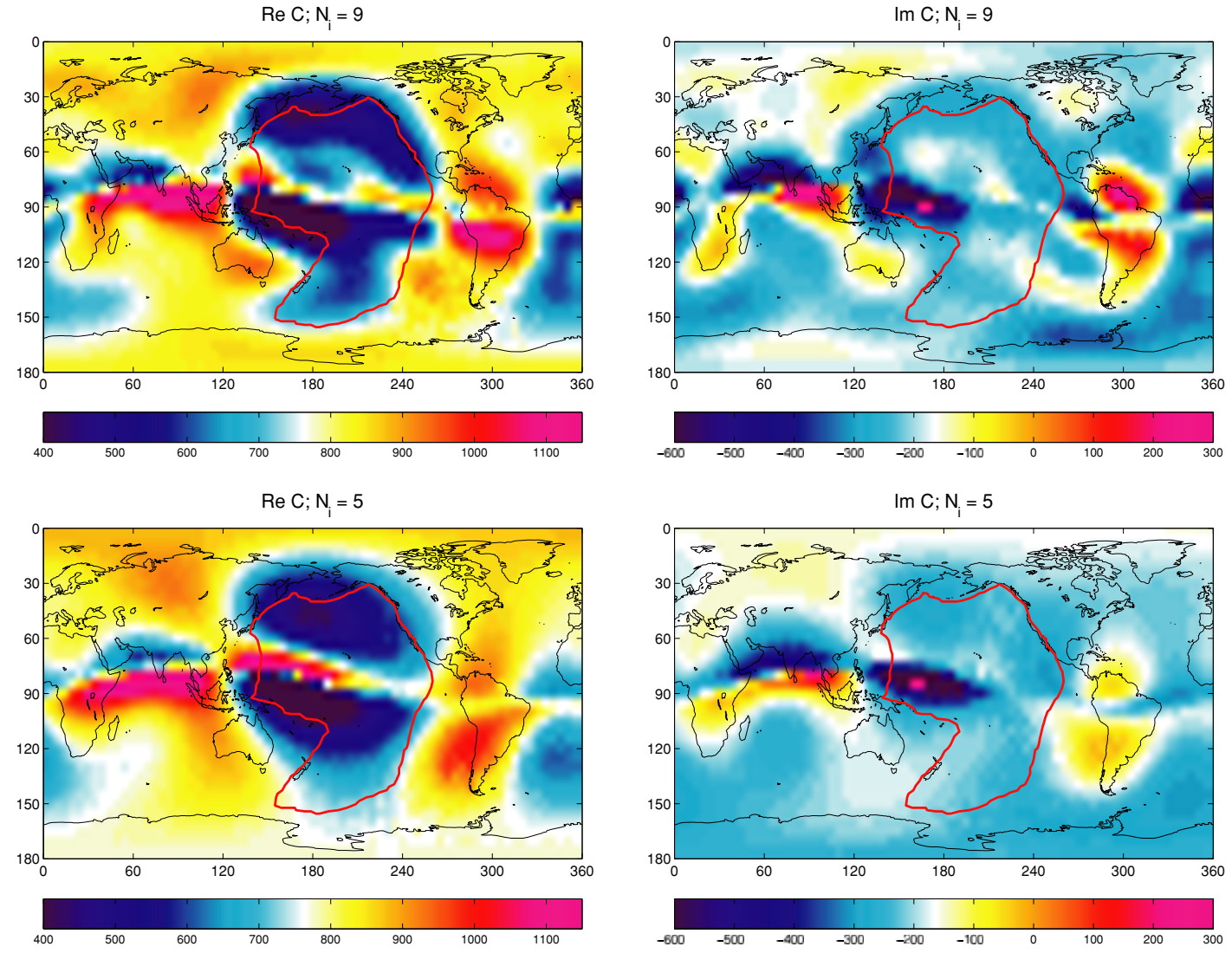

Fig. 6. Real (left panels) and imaginary (right panels) parts of $C$-responses at period of 7.8 days, obtained with $N_{i}=9$ (upper panels) and $N_{i}=5$ (lower panels), respectively.
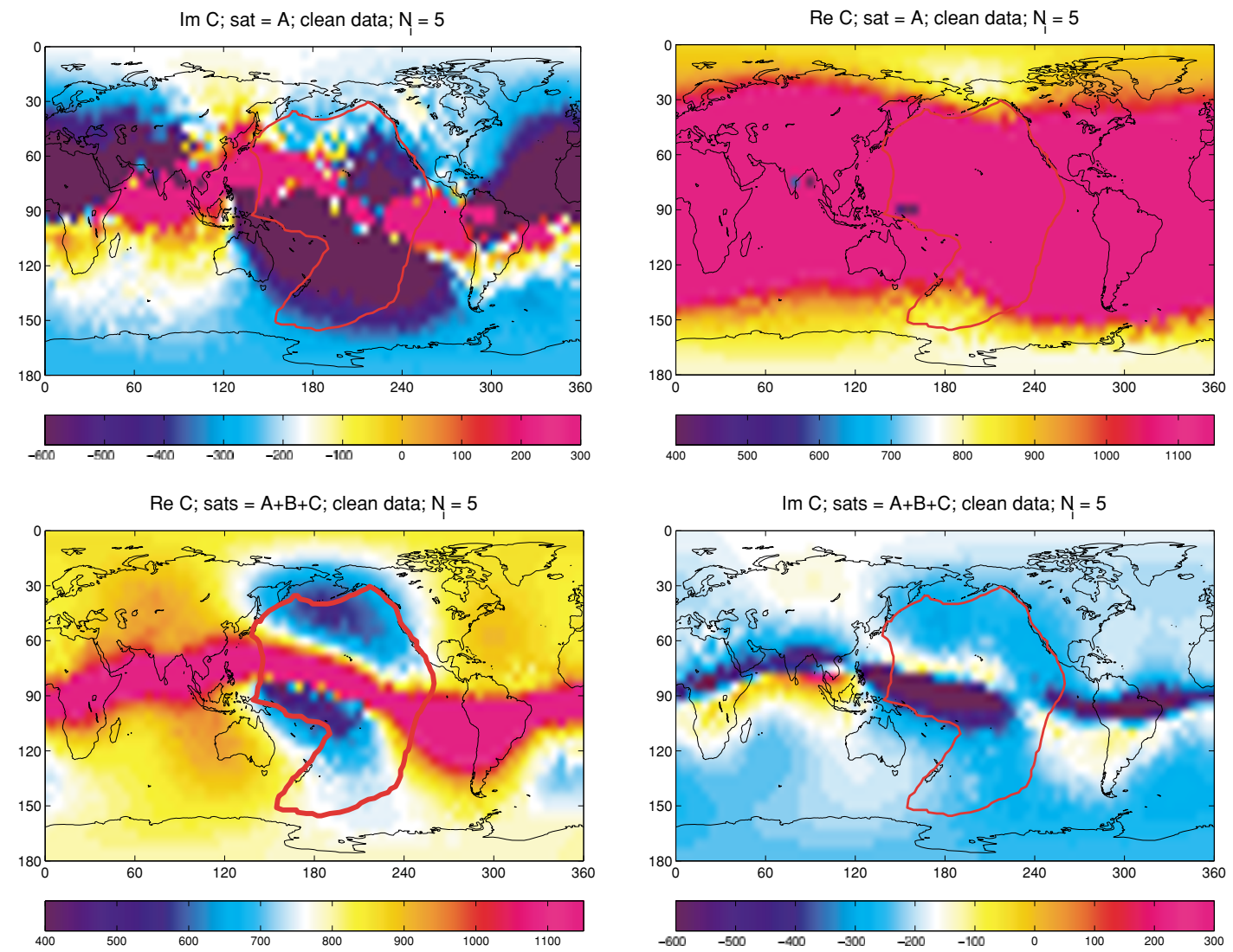

Fig. 7. Real (left panels) and imaginary (right panels) parts of $C$-responses at period of 7.8 days, which were estimated from satellite data that contain only magnetospheric contribution. Upper and lower panels present respectively the results when single satellite (A) data and data from constellation of satellites $(\mathrm{A}+\mathrm{B}+\mathrm{C})$ were used for recovery (with $N_{i}=5, \Delta_{C}=6$ hours). 
Let magnetic signals from magnetospheric sources, $\left(B_{r}^{(s)}, B_{\vartheta}^{(s)}, B_{\varphi}^{(s)}\right)$ at time instant $t_{i}$ and position $\left(r_{i}^{(s)}, \vartheta_{i}^{(s)}, \varphi_{i}^{(s)}\right)$, be provided by a constellation of satellites. Here $t_{i}=i \Delta_{D}, i=1,2, \ldots, N_{D}, s=1,2, \ldots, N_{S}$, where $\Delta_{D}$ is sampling interval, and $N_{D}$ and $N_{S}$ are the number of samples and satellites, respectively.

Assuming that no electric currents exist at satellite altitude, the magnetic field can be derived from a scalar magnetic potential. It means, in particular, that one can in principle reconstruct time series (with some sampling interval, $\Delta_{C}$ ) of the external and induced coefficients from magnetic signals using a least-square approach. Note that this scheme of reconstruction of the coefficients has been successfully applied by Olsen et al. (2002) to calculate global 1-D response. Once the coefficients, $q_{n}^{m}(t), s_{n}^{m}(t)$ and $g_{k}^{l}(t), h_{k}^{l}(t)$, have been determined, time series of $B_{r}$ and $\nabla_{H} \cdot \mathbf{B}_{H}$ (with the same sampling interval $\Delta_{C}$ ) are reconstructed on a regular grid at the surface of the Earth by spherical harmonic synthesis. Signal processing of $B_{r}$ and $\nabla_{H} \cdot \mathbf{B}_{H}$ allows for an estimation of $C(\omega, r, \vartheta, \varphi)$ using Eq. (3).

Figure 7 presents maps of the $C$-response for a period of 7.8 days obtained using data from one satellite (A; upper panel) and from a constellation of three satellites $(A+B+C$; lower panel), respectively. The details of the orbit evolutions of satellites A, B and C are discussed in the companion paper of Olsen et al. (2006). We used data of 1 min sampling interval (experiments with $5 \mathrm{sec}$ sampling interval did not improve the results). The sampling interval of the resulting coefficients, the length of the time series, and the number of internal coefficients were chosen to be 6 hours, 3 years (1999-2002) and $35\left(N_{i}=5\right)$, respectively. It is clearly seen that only a constellation allows for the recovery of the $C$-responses. From comparing the lower panels of Figs. 6 and 7 one can conclude that the recovered $C$-responses are consistent in geometry with the true $C$-responses. The key is the availability of simultaneous observations of the magnetic field variation at different local times, i.e. a spatio-temporal coverage sufficient to reproduce the magnetic fields induced by magnetospheric source.

Figure 8 confirms this quantitatively. Shown are the relative differences, $\left|C^{(r)}-C^{(t)}\right| /\left|C^{(t)}\right|$, between the recovered, $C^{(r)}$, and the true responses, $C^{(t)}$, in the period range between 4 and 15 days for the same three sites that were used in Section 4. The differences (i.e., the relative error) are about $20 \%$ for the North Pacific Ocean site, and about $15 \%$ for the sites located in the South Indian Ocean and in Central Eurasia. For comparison, relative differences are presented for the case of response estimation using single satellite data. In that case the relative differences are unsatisfactory large, exceeding $100 \%$ at a period of 4 days and still remaining about $30 \%$ at a period of 15 days. The results obtained using single satellite data are closer to those from multiple satellites data as the period increases because the 3-D effect in $C$-responses weakens at longer periods. Thus they are more 1-D like, a situation that can be treated using single satellite data. It should be especially noted that even for the case when data from a constellation have been used the relative difference between recovered and true responses is $15-20 \%$. This is most likely due to the fact that the time series of spherical harmonic coefficients (es- pecially of higher orders) could not be recovered without some alising, due to the complicated spatio-temporal characteristics of satellite data.

Next we attempt to improve the image of the deep conductor in the $C$-responses by using more internal coefficients for description of $B_{r}(t)$ and $\nabla_{H} \cdot \mathbf{B}_{H}(t)$. However there is a counterbalance between the number of coefficients to be resolved, and the sampling interval of the resulting series. If we want to resolve coefficients with higher degree $k$ and order $l$, we have to choose a longer sampling interval, $\Delta_{C}$, in order to provide sufficient spatial coverage of the satellite data. So in this series of calculations the sampling interval was chosen to be 12 hours (instead of 6 hours of the previous example) and the number of internal coefficients was $99\left(N_{i}=9\right)$ with the same length (3 years; 1999-2002 period) of the time series analysed. Figure 9 shows the $C$-responses at a period of 8 days (the slight shift in the period compared to the previous results is due to different sampling interval). The geometry of the anomaly in South Pacific Ocean is more clearly identified with these new sampling interval and number of coefficients (cf. lower panels on Fig. 7). Choosing the old sampling interval of 6 hours with the new (larger) number of internal coefficients resulted in $C$-responses (not shown here) that are worse compared to the case of 12 hours sampling interval.

\section{7. $C$-responses from Satellite Data that Contain All Modelled Contributions}

Finally we demonstrate the possibility to recover $C$ responses from satellite data that contain, along with the magnetospheric signals, realistic contributions from all other sources modelled - from core and crustal fields, ionospheric variations, as well as payload noise (see details of the forward modelling of these contributions in the companion paper of Olsen et al. (2006)). Time series of the coefficients $q_{n}^{m}(t), s_{n}^{m}(t)$ and $g_{k}^{l}(t), h_{k}^{l}(t)$ were obtained using Comprehensive Inversion, as described in the companion paper of Sabaka and Olsen (2006). The sampling interval, the length of the time series, and the number of internal coefficients were chosen to be 6 hours, 3 years (1999-2002) and 35 (with $N_{i}=5$ ), respectively, and three satellites $($ Swarm $A+B+C)$ have been used for the recovery. Figure 10 shows maps of the recovered $C$-responses at a period of 7.8 days. It is remarkable that the results happened to be in rather good agreement with those obtained from the satellite data that contain only magnetospheric field (cf. lower panels on Fig. 7).

\section{Conclusions}

In this paper we present an approach to detect large-scale conductivity anomalies deeply embeddeded in the mantle by analysis of magnetic signals from low-Earth-orbiting satellites. The approach deals with recovery of $C$-responses on a regular spatial grid and starts with a determination of time series of external and internal coefficients of the magnetic potential. From the coefficients, time series of magnetic vertical component and the horizontal derivatives of the horizontal components are synthesized on the grid and the $C$-responses are determined by means of signal 


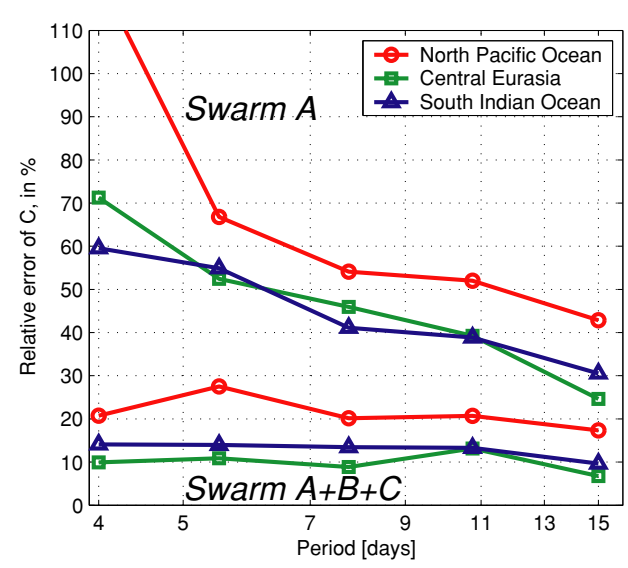

Fig. 8. Relative difference, $\left|C^{(r)}-C^{(t)}\right| /\left|C^{(t)}\right|$, in $\%$, between recovered, $C^{(r)}$, and true responses, $C^{(t)}$, for the period range from 4 to 15 days for three sites with essentially different conductivity vs. depth profiles beneath the sites. Abbreviations "Swarm A" and "Swarm A+B+C" stand for respective cases when data from single satellite (A) and from constellation of satellites $(\mathrm{A}+\mathrm{B}+\mathrm{C})$ were used for recovery (with $N_{i}=5$, $\Delta_{C}=6$ hours).

processing of the corresponding time series.

We demonstrate that global maps of $C$-responses are successfully recovered from magnetic data collected by the proposed Swarm constellation. For this demonstration we use synthetic magnetic signals from a given realistic magnetospheric source distribution and realistic 3-D conductivity model. To simulate the signals we apply an integral equation method to solve Maxwell's equations in the frequency domain and then obtain time-domain solutions by means of an inverse Fourier transform.

We identify several areas where the developed recovery scheme could be improved. Possible topics for future research include a more accurate calculation of the external and internal coefficients by using a robust least square approach with constraints, and utilization of improved signal processing methods (cf. Riedel and Sidorenko, 1995).

Eventually, the $C$-responses recovered on a regularly spaced grid at a set of periods could serve as input for a rigorous 3-D inversion, yet to be developed, in order to retrieve 3-D models of the electrical conductivity distribution in the Earth's mantle.

The $C$-response approach described in this paper is only one of several possible ways to attack the 3-D satellite induction problem. The complicated spatio-temporal characteristics of satellite data may favour the application of timedomain techniques (cf. Everett and Martinec, 2003; Martinec and McCreadie, 2004; Velimsky et al., 2006; Kuvshinov and Olsen, 2005a). For example there is a hope that with the use of time-domain approach it would be possible to resolve upper mantle local conductors that we failed to see in global maps of $C$-responses.

Acknowledgments. ESA funded this study through contract No. $17263 / 03 /$ NL/CB. This work is supported in part by the Russian Foundation for Basic Research under grant No. 03-05-64656.
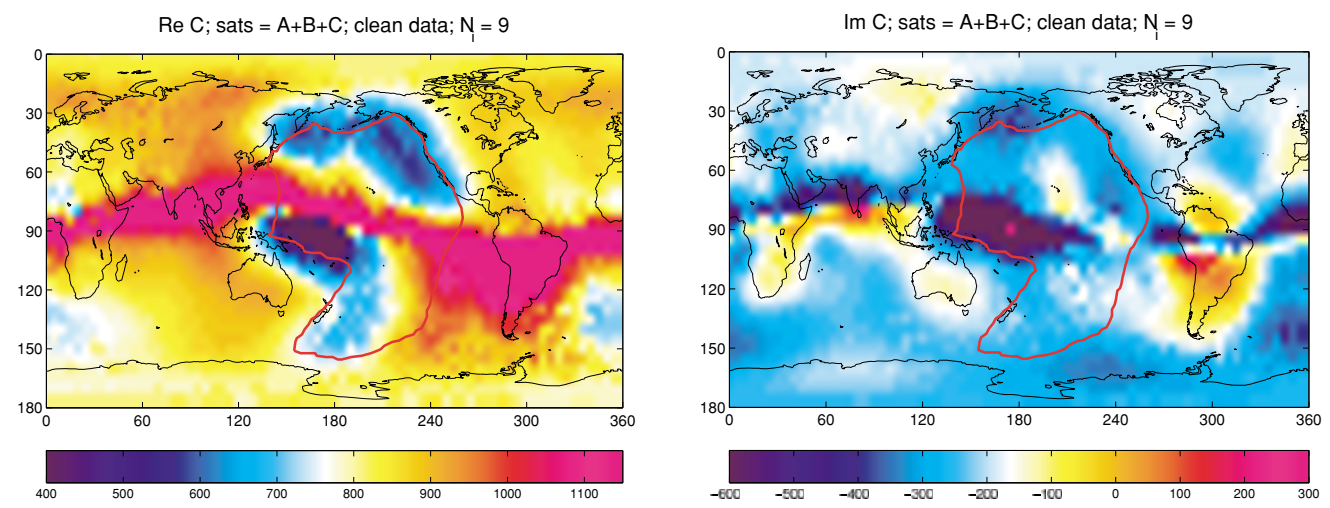

Fig. 9. Real (left panel) and imaginary (right panel) parts of $C$-responses at period of 8 days, which were estimated from constellation $(\mathrm{A}+\mathrm{B}+\mathrm{C})$ data that contain only magnetospheric contribution (with $N_{i}=9, \Delta_{C}=12$ hours).
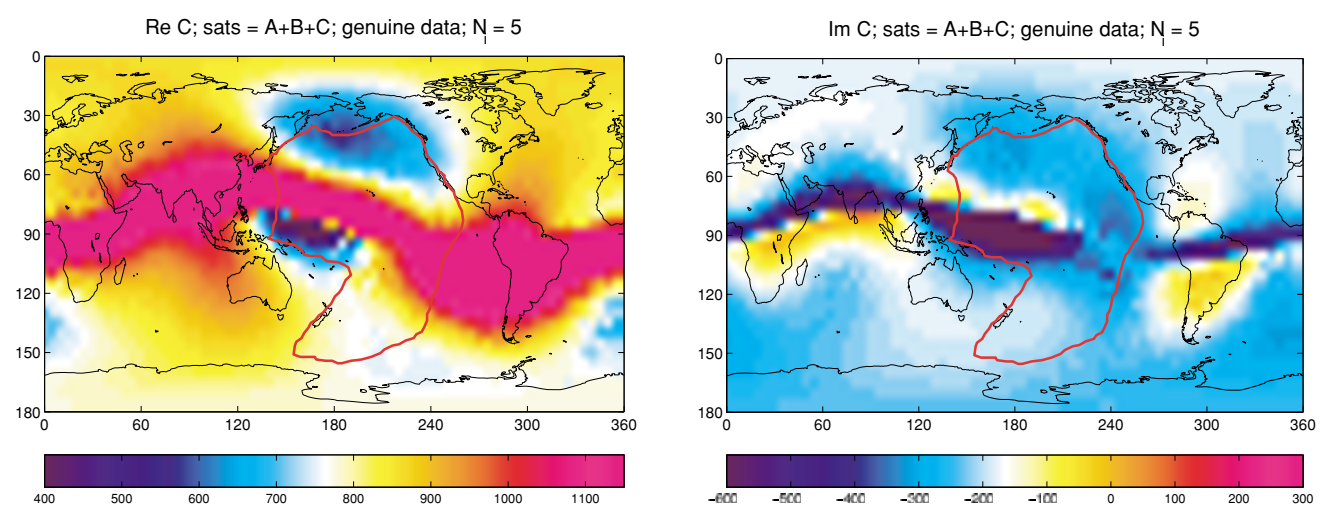

Fig. 10. Real (left panel) and imaginary (right panel) parts of $C$-responses at period of 7.8 days, which were estimated from constellation $(\mathrm{A}+\mathrm{B}+\mathrm{C})$ data that contain all modelled contributions (with $N_{i}=5, \Delta_{C}=6$ hours). 


\section{References}

Bijwaard, H. and W. Spakman, Non-linear global P-wave tomography by iterated linearized inversion, Geophys. J. Int., 110, 251-266, 2000.

Constable, S. and C. Constable, Observing geomagnetic induction in magnetic satellite measurements and associated implications for mantle conductivity, Geochemistry, Geophysics, Geosystems, 5, doi:10.1029/ 2003GC000,634, 2004

Constable, S. and G. Heinson, Hawaiian hot-spot swell structure from seafloor MT sounding, Tectonophysics, 389, 111-124, 2004.

Deschamps, F., J. Trampert, and R. Snieder, Anomalies of temperature and iron in the uppermost mantle inferred from gravity data and tomographic models, Phys. Earth Planet. Int., 129, 245-264, 2002.

Didwall, E. M., The electrical conductivity of the upper mantle as estimated from satellite magnetic field data, J. Geophys. Res., 89, 537-542, 1984.

Egbert, G. D. and J. R. Booker, Very long period magnetotellurics at Tucson observatory: implication for mantle conductivity, J. Geophys. Res., 97, 15,099-15,112, 1992

Everett, M. and Z. Martinec, Spatiotemporal response of a conducting sphere under simulated geomagnetic storm conditions, Phys. Earth Planet. Int., 138, 163-181, 2003.

Everett, M., S. Constable, and C. Constable, Effects of near-surface conductance on global satellite induction responses, Geophys. J. Int., 153, 277-286, 2003.

Fujii, I. and H. Utada, On geoelectric potential variations over a planetary scale, Memoirs of the Kakioka Magnetic Observatory, 29, 1-81, 2000.

Fukao, Y., T. Koyama, M. Obayashi, and H. Utada, Trans-Pacific temperature field in the mantle transition region derived from seismic and electromagnetic tomography, Earth and Planetary Science Letters, 217, 425-434, 2004.

Grammatica, N. and P. Tarits, Contribution at satellite altitude of electromagnetically induced anomalies arising from a three-dimensional heterogeneously conducting earth, using $\mathrm{Sq}$ as an inducing source field, Geophys. J. Int., 151, 913-923, 2002.

Kuvshinov, A. and N. Olsen, Mantle conductivity obtained by 3-D inversion of magnetic satellite data - an approach and its validation, Geophysical Research Abstracts, 7, 08,607, 2005 a.

Kuvshinov, A. and N. Olsen, Modelling the ocean effect of geomagnetic storms at ground and satellite altitude, in Earth Observation with CHAMP. Results from Three Years in Orbit, edited by C. Reigber, H. Lühr, P. Schwintzer, and J. Wickert, pp. 353-359, Springer Verlag, 2005 b

Kuvshinov, A., D. Avdeev, and O. Pankratov, On deep sounding of a nonhomogeneous earth using satellite magnetic measurements, Physics of the Solid Earth, 34, 326-331, 1998.

Kuvshinov, A. V., D. B. Avdeev, O. V. Pankratov, S. A. Golyshev, and N. Olsen, Modelling electromagnetic fields in 3D spherical Earth using fast integral equation approach, in 3 D Electromagnetics, edited by M. S. Zhdanov and P. E. Wannamaker, chap. 3, pp. 43-54, Elsevier, Holland, 2002.

Kuvshinov, A. V., H. Utada, D. Avdeev, and T. Koyama, 3-D modelling and analysis of Dst C-responses in the North Pacific ocean region, revisited, Geophys. J. Int., 160, 505-526, 2005.

Laske, G. and G. Masters, A global digital map of sediment thickness, EOS Trans. AGU, 78, F483, 1997.

Li, X. D. and B. Romanowicz, Global mantle shear velocity model developed using nonlinear asymptotic coupling theory, J. Geophys. Res., 101, 22,245-22,272, 1995.

Lizarralde, D., A. Chave, G. Hirth, and A. Schultz, Long period magnetotelluric study using Havaii-to-California submarine cable data: implications for mantle conductivity, J. Geophys. Res., 100, 17,873-17,884, 1995.

Martinec, Z. and H. McCreadie, Electromagnetic induction modelling based on satellite magnetic vector data, Geophys. J. Int., 155, 33-43, 2004.

McCreadie, H. and Z. Martinec, Geomagnetic induction modelling based on CHAMP magnetic vector data, in Earth Observation with CHAMP Results from Three Years in Orbit, edited by C. Reigber, H. Lühr, P. Schwintzer, and J. Wickert, pp. 335-341, Springer Verlag, 2005.

Neal, S. L., R. L. Mackie, J. C. Larsen, and A. Schultz, Variations in the electrical conductivity of the upper mantle beneath North America and the Pacific Ocean, J. Geophys. Res., 105, 8229-8242, 2000.

Olsen, N., Estimation of $C$-responses $(3 \mathrm{~h}$ to $720 \mathrm{~h}$ ) and the electrical conductivity of the mantle beneath Europe, Geophys. J. Int., 133, 298-
308,1998

Olsen, N., Induction studies with satellite data, Surveys in Geophysics, $\mathbf{2 0}$ 309-340, 1999.

Olsen, N. and A. Kuvshinov, Modelling the ocean effect of geomagnetic storms, Earth Planets Space, 56, 525-530, 2004.

Olsen, N., S. Vennerstrøm, and E. Friis-Christensen, Monitoring magnetospheric contributions using ground-based and satellite magnetic data, in First CHAMP Mission Results for Gravity, Magnetic and Atmospheric Studies, edited by C. Reigber, H. Lühr, and P. Schwintzer, pp. 245-250, Springer Verlag, 2002.

Olsen, N., R. Haagmans, T. J. Sabaka, A. Kuvshinov, S. Maus, M. E. Purucker, M. Rother, V. Lesur, and M. Mandea, The Swarm End-to-End mission simulator study: A demonstration of separating the various contributions to Earth's magnetic field using synthetic data, Earth Planets Space, 58, this issue, 359-370, 2006.

Oraevsky, V. N., N. M. Rotanova, T. N. Bondar, D. Y. Abramova, and V. Y. Semenov, On the radial geoelectrical structure of the mid-mantle from magnetovariational sounding using MAGSAT data, J. Geomag. Geoelectr., 45, 1415-1423, 1993.

Riedel, K. and A. Sidorenko, Minimum bias multiple taper spectral estimation, IEEE Trans. Signal Process., 43, 188-195, 1995.

Ritsema, J., H. J. van Heijst, and J. H. Woodhouse, Complex shear velocity structure imaged beneath Africa and Iceland, Science, 286, 1925-1928, 1999.

Roberts, R. G., The long-period electromagnetic response of the earth, Geophys. J. R. astr. Soc., 78, 547-572, 1984.

Sabaka, T. J. and N. Olsen, Enhancing comprehensive inversions using the Swarm constellation, Earth Planets Space, 58, this issue, 371-395, 2006.

Santos, F., A. Soares, H. Rodriges, R. Luzio, and N. Palshin, Lithosphere conductivity structure using the CAM-1 (Lisbon-Madeira) submarine cable, Geophys. J. Int., 155, 591-600, 2003.

Schmucker, U., Magnetic and electric fields due to electromagnetic induction by external sources, in Landolt-Börnstein, New-Series, $5 / 2 b$, pp. 100-125, Springer-Verlag, Berlin-Heidelberg, 1985.

Schultz, A., On the vertical gradient and associated heterogeneity in mantle electrical conductivity, Phys. Earth Planet. Interiors, 64, 68-86, 1990.

Schultz, A. and J. C. Larsen, On the electrical conductivity of the midmantle-I. Calculation of equivalent scalar magnetotelluric response functions, Geophys. J. R. astr. Soc., 88, 733-761, 1987.

Schultz, A., R. Kurtz, A. Chave, and A. Jones, Conductivity discontinuities in the upper mantle beneath a stable craton, Geophys. Res. Lett., 20, 2941-2944, 1993

Semenov, V., W. Jozwiak, and J. Pek, Deep electromagnetic soundings conducted in Trans-European Suture Zone, EOS Trans. AGU, 84, 581, 2003.

Su, W. J. and A. M. Dziewonski, Simultaneous inversions for 3-D variations in shear and bulk velocity in the mantle, Phys. Earth Planet. Int., 100, 135-156, 1997.

Tarits, P. and N. Grammatica, Electromagnetic induction effects by the solar quiet magnetic field at satellite altitude, Geophys. Res. Lett., 27, 4009-4012, 2000

Utada, H., T. Koyama, H. Shimizu, and A. D. Chave, A semi-global reference model for electrical conductivity in the mid-mantle beneath the North Pacific region, Geophys. Res. Lett., 30, doi:10.1029/ 2002GL016,092, 2003.

Velimsky, J. and M. Everett, Electromagnetic induction by Sq ionospheric currents in a heterogeneous Earth: Modeling using ground-based and satellite measurements, in Earth Observation with CHAMP. Results from Three Years in Orbit, edited by C. Reigber, H. Lühr, P. Schwintzer, and J. Wickert, pp. 341-347, Springer Verlag, 2005.

Velimsky, J., M. E. Everett, and Z. Martinec, The transient Dst electromagnetic induction signal at satellite altitudes for a realistic 3-D electrical conductivity in the crust and mantle, Geophys. Res. Lett., 30, doi:10.1029/2002GL016,671, 2003.

Velimsky, J., Z. Martinec, and M. Everett, Electrical conductivity in the Earth's mantle inferred from CHAMP satellite measurements-I. Data processing and 1-D inversion, Geophys. J. Int., 2006 (in press).

Woodhouse, J. and J. Trampert, Global upper mantle structure inferred from surface wave and body wave data, EOS Trans., p. F422, 1995.

A. Kuvshinov (e-mail: alexei@spacecenter.dk), T. Sabaka, and N. Olsen 